\title{
Délos : étude de morphologie urbaine - 2018
}

Philippe Fraisse et Lionel Fadin

\section{OpenEdition \\ Journals}

Édition électronique

URL : http://journals.openedition.org/baefe/1917

DOI : $10.4000 /$ baefe. 1917

ISSN : 2732-687X

Éditeur

ResEFE

\section{Référence électronique}

Philippe Fraisse, Lionel Fadin, « Délos : étude de morphologie urbaine - 2018 » [notice archéologique], Bulletin archéologique des Écoles françaises à l'étranger [En ligne], Grèce, mis en ligne le 23 décembre 2020, consulté le 22 mars 2021. URL : http://journals.openedition.org/baefe/1917 ; DOI : https:// doi.org/10.4000/baefe.1917

Ce document a été généré automatiquement le 22 mars 2021.

\section{cc)}

Le Bulletin archéologique des Écoles françaises à l'étranger est mise à disposition selon les termes de la Licence Creative Commons Attribution - Pas d'Utilisation Commerciale - Pas de Modification 4.0 International. 


\title{
Délos : étude de morphologie urbaine - 2018
}

\author{
Philippe Fraisse et Lionel Fadin
}

\section{NOTE DE L'AUTEUR}

Autorité nationale présente : Éphorie des Cyclades

Numéro de mission : C36

Établissements porteurs de l'opération : EFA

\author{
Données scientifiques produites \\ Délos par l'EFA \\ Web SIG de Délos
}

1 Cette année encore les activités de terrain liées à l'exploration des secteurs urbains non fouillés, qui a été initiée en 2013, se sont poursuivies; elles ont été suivies d'une phase de traitement des nouvelles données recueillies dans la région située au Nord du Quartier du Stade.

2 La campagne sur le terrain avait un double objectif.

3 Il s'agissait en premier lieu d'abonder le relevé topographique du secteur situé au Nord du Stade, avec les vestiges qui avaient pu nous échapper lors des opérations de terrain de 2014, mais qui apparaissaient de façon plus nette sur l'orthophotoplan que nous avions réalisé en 2017. Grâce à l'apport de ces informations nouvelles, les données figurant sur le plan topographique existant ont pu être complétées avec un certain nombre de structures supplémentaires. Il est à noter que si la connaissance de ce secteur a pu ainsi être enrichie, pour autant, la découverte de ces nouveaux éléments ne remet pas en question les hypothèses de restitution que nous avions déjà pu avancer. 
4 L'article rédigé à l'issue de cette campagne présente l'ensemble des relevés topographiques qui ont été réalisés dans cette zone, et offre une première synthèse des acquis de l'étude conduite au nord du Stade, sur le secteur de la presqu'île de Patinioti. Les hypothèses que nous avons pu élaborer sur les modalités d'urbanisation de cette région, à partir de l'interprétation des structures repérées, y sont présentées en détail. De même, les différentes logiques qui ont présidé à l'implantation du bâti, selon deux processus d'urbanisation distincts, dans la bande littorale, ou plus à l'intérieur des terres, et les règles qui semblent les avoir accompagnées, sont exposées et largement commentées.

5 Le survol par un drone de la région explorée en 2015, constituait le second objectif de cette mission. Cette zone correspond à un vaste territoire incluant la colline de Glastropi, qui va des abords Sud de la Maison des Masques jusqu'à la côte occidentale. À l'instar de ce qui avait été fait au Nord du Stade, il s'agissait de prendre les prises de vue nécessaires à la réalisation d'un orthophotoplan. L'intérêt que présente ce type de vues afin non seulement de vérifier les relevés topographiques faits depuis le sol, mais également de compléter éventuellement la collecte des données de terrain, n'est plus à démontrer. Nous nous sommes donc fixé pour but de réaliser cette opération sur la totalité des zones urbanisées non fouillées à Délos.

6 Les vols de drone auxquels nous avons pu procéder ont permis d'obtenir une couverture photographique complète des structures archéologiques visibles en surface du terrain naturel. Les calculs nécessaires pour la réalisation de cette vue orthonormée devraient aboutir au cours de cet automne, et nous fournir une représentation en 3D du secteur concerné. La superposition de cette image avec le plan des vestiges dessinés lors de la campagne de relevés topographiques de 2015, devrait faciliter le travail d'interprétation des structures présentes dans une région à la topographie mouvementée.

7 La zone située immédiatement au Sud de la maison des Masques présente une physionomie particulière. Les éléments naturels, comme notamment les dépressions plus ou moins profondes et plus ou moins vastes qui caractérisent le relief, semblent avoir guidé l'implantation de grosses demeures assez semblables à la maison des Masques (Fig. 1). Ces cavités devaient, à certaines périodes de l'année, se charger en eau, fournissant à leurs habitants les réserves dont ils avaient besoin pour un usage quotidien. 
Fig.1. Dépression dans le rocher au Sud de la Maison des Masques.

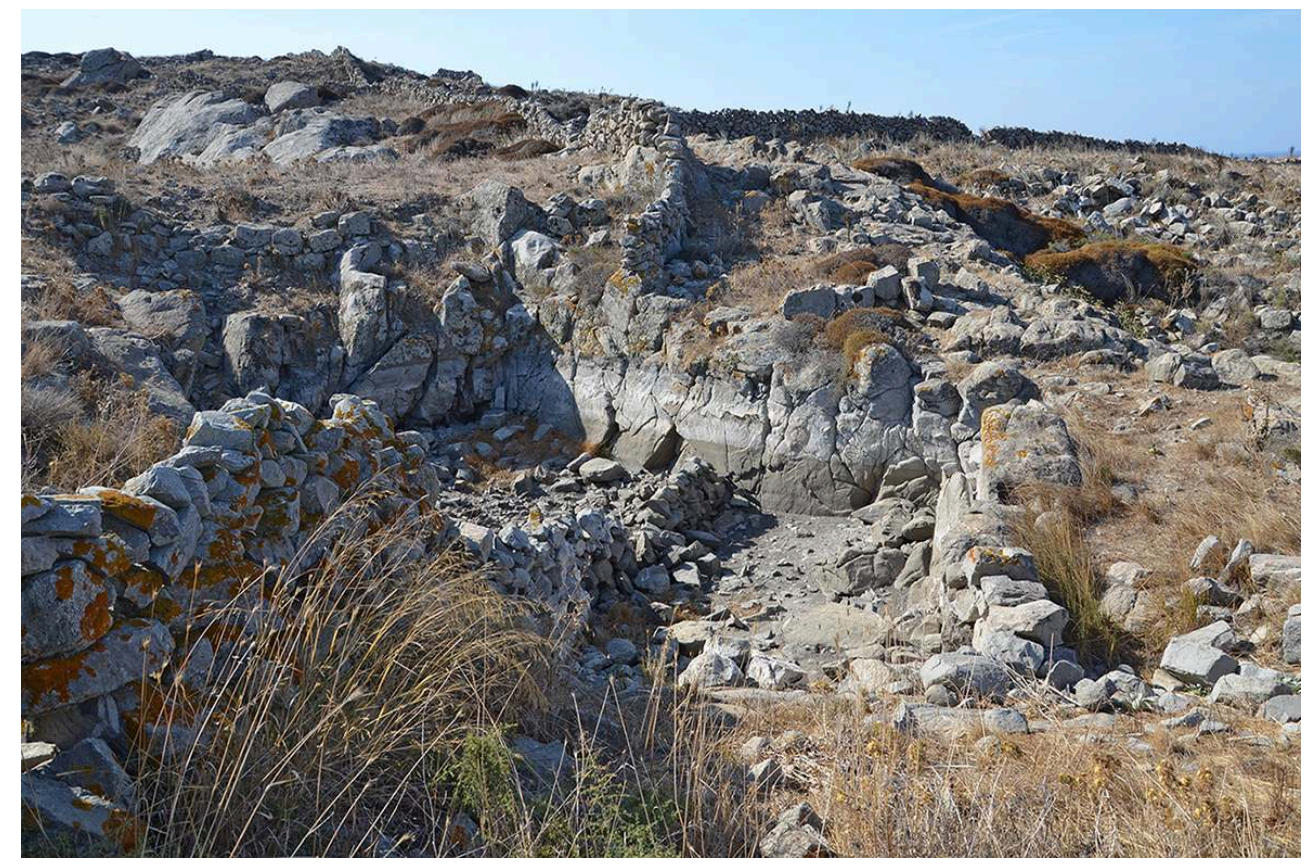

EFA.

Fig.2. Vestiges du mur Nord de l'édifice construit au sommet de la colline de Glastropi.

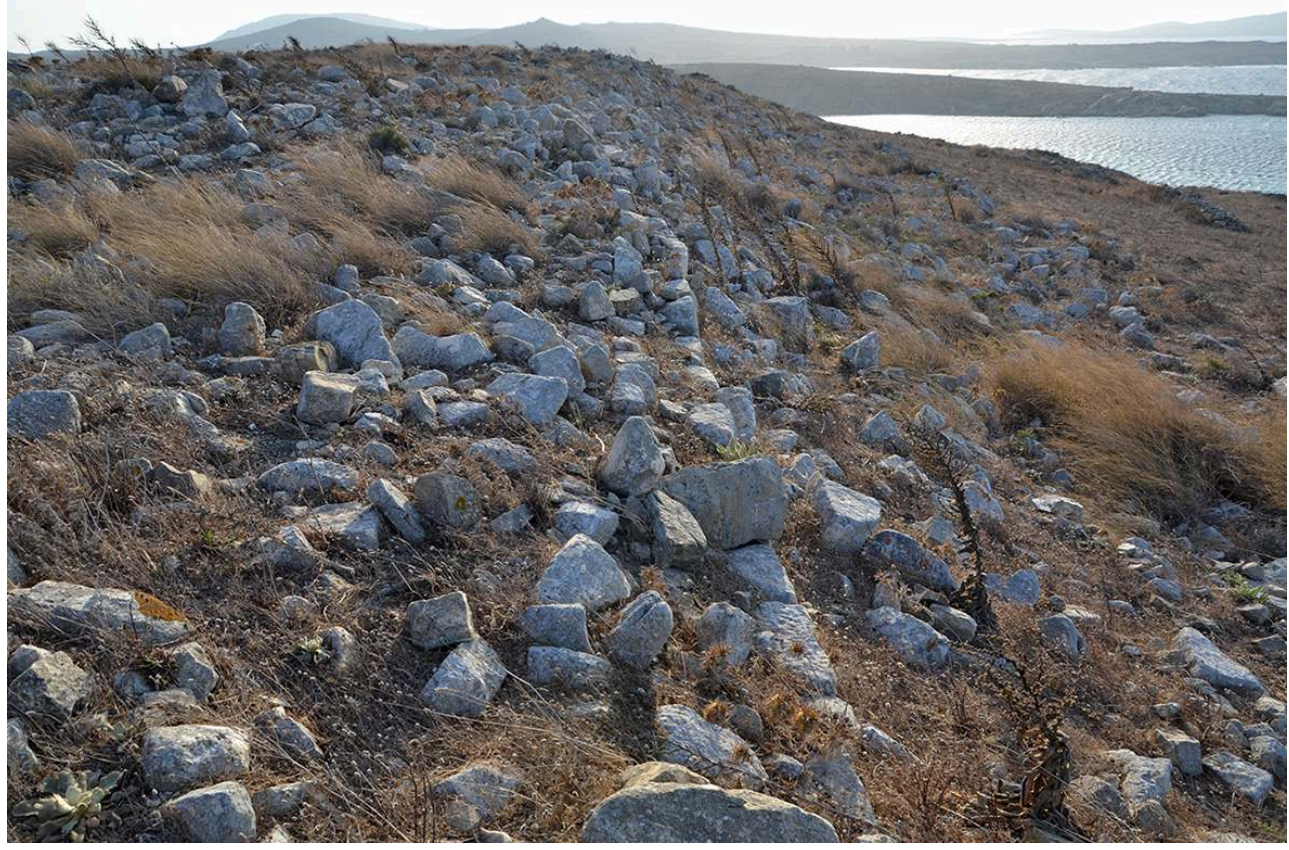

EFA.

8 Au sommet de la colline de Glastropi, on observe les vestiges de murs construits en marbre, qui appartenaient à un édifice dont le plan n'a pas d'équivalent à Délos. Peu profond, il présente une longueur de près d'une centaine de mètres, ainsi qu'une certaine régularité dans sa partition. On observe une série de pièces aux dimensions voisines, séparées entre elles par de longs couloirs qui semblent être des circulations 
intérieures (Fig. 2). L'originalité de son plan et l'utilisation du marbre comme matériau de construction justifient à elles seules une étude architecturale de cet édifice.

Plus à l'ouest, l'urbanisation s'est développée dans la pente jusqu'à la falaise côtière qui surplombe la mer.

Comme dans le cas des travaux menés au Nord de l'île, l'exploitation des photos aériennes obligera sans doute à un retour sur le terrain, afin de compléter le relevé topographique des quelques structures non visibles in situ, mais identifiables sur les images prises par le drone. Cette tâche une fois menée à son terme, il sera possible d'entreprendre une réflexion qui nous permette d'élaborer des hypothèses de restitution de la forme urbaine qui s'était développée dans cette région, et d'en dégager les traits particuliers.

D'ores et déjà, les observations auxquelles nous nous sommes livrés, nous laissent penser que l'urbanisation de cette zone s'est faite selon des modalités assez différentes de celles que nous avons pu restituer dans la région au Nord du Stade, puisqu'ici la trame urbaine ne paraît pas organisée en îlots. Même si le réseau des voies desservant le bâti apparaît ici de manière beaucoup moins lisible, il fut sans doute beaucoup moins développé, et ne répondait pas aux principes suivis dans le quartier du Théâtre. On a semble-t-il affaire à un quartier limitrophe de la ville, où le bâti est loin d'être aussi homogène, et où les caractéristiques naturelles de la topographie semblent avoir joué un rôle majeur dans l'implantation des constructions.

\section{INDEX}

chronologie https://ark.frantiq.fr/ark:/26678/pcrtNzYn31IIAZ

Thèmes : EFA

sujets 26678/pcrtKJVpuP3AET, https://ark.frantiq.fr/ark:/26678/crtKTzSReth5I, https://

ark.frantiq.fr/ark:/26678/pcrtaodMT8j830

Année de l'opération : 2018

lieux https://ark.frantiq.fr/ark:/26678/pcrtXvsaqTQa4J

\section{AUTEURS}

\section{PHILIPPE FRAISSE}

École française d'Athènes

\section{LIONEL FADIN}

École française d'Athènes 